\title{
The Intramuscular Activation of Scapular Stabilizing Muscles during Push-up plus and PNF Exercises in a Quadruped Position
}

\author{
Du-Jin Park, MS, PT ${ }^{1)}$, Hyun-Ok Lee, PhD, $\left.\mathrm{PT}^{2}\right)^{*}$ \\ 1) Department of Physical Therapy, Graduate School of Life Sciences, Catholic University of Pusan \\ 2) Department of Physical Therapy, College of Health Sciences, Catholic University of Pusan: \\ 9 Bugok, 3-dong, Geumjung-gu, Busan 609-757, Republic of Korea. TEL: +82 51-510-0570, \\ FAX: +82 51-510-0578
}

\begin{abstract}
Purpose] The purpose of this study was to identify the upper trapezius/serratus anterior (UT/SA) ratio and the intramuscular activation of scapular stabilizing muscles during proprioceptive neuromuscular facilitation (PNF) and push-up plus (PUP) exercises in a quadruped position. [Subjects and Methods] Fourteen healthy men voluntarily participated as research subjects for this experiment. All subjects were measured in terms of the activation of their scapular stabilizing muscles using surface electromyography during random PNF and PUP exercises in a quadruped position. [Results] The PUP exercise showed a significantly higher level of middle serratus anterior activity than the PNF exercise. The PNF exercise showed statistically significant higher level of lower trapezius and lower serratus anterior activities than the PUP exercise. There was a significantly lower UT/LSA ratio in the PNF than in the PUP exercise. [Conclusion] The PUP exercise is more suitable for middle serratus anterior training than the PNF exercise. Furthermore, the PNF exercise is more effective than the PUP exercise as a training method for lower trapezius and lower serratus muscles and an unbalanced UT/LSA ratio.

Key words: PNF, Push-up plus, Scapular stabilizing muscle
\end{abstract}

(This article was submitted Oct. 19, 2012, and was accepted Nov. 7, 2012)

\section{INTRODUCTION}

The serratus anterior (SA), the first stabilizing muscle of the scapulothoracic articulation, plays an important role in stabilizing the medial scapular in the thorax and provides dynamic stability to the scapulothoracic movement ${ }^{1,2)}$. The SA is the sole muscle that contributes to normal threedimensional scapular movements in relation to the thorax during arm elevation ${ }^{3)}$. The stability and movement of the scapular, particularly while an individual performs a task above the head with the arms raised, are both closely related to SA activity ${ }^{4}$.

Abnormal SA function is associated with abnormal scapular movement ${ }^{5)}$ and can cause shoulder joint problems such as the shoulder instability of throwing athletes ${ }^{6}$, the shoulder pain of swimmers ${ }^{7}$ ) and shoulder impingement syndrome ${ }^{5,8)}$. Consequently, individuals with shoulder problems increase the activity of their upper trapezius (UT) to compensate for the decreased activity of the SA, which, in turn, decreases the upward rotation and posterior tipping of the scapular ${ }^{8)}$. Therefore, training that can reduce the UT activity while simultaneously strengthening the SA is essential. For this reason, some researchers have identified various shoulder training effects using the UT/SA ratio ${ }^{9-11)}$

Among the various shoulder rehabilitation exercises, the

*To whom correspondence should be addressed.

E-mail: holee@cup.ac.kr push-up plus (PUP) is effective in minimizing the UT activity and increasing the SA activity ${ }^{9,10)}$. However, Lunden et al. ${ }^{12)}$ noted that scapulothoracic and glenohumeral movements during the PUP exercise can decrease the subacromial space and induce impingement of the rotator cuff. In addition, since the PUP is performed while the arms are raised up to $90^{\circ}$, it could have limitations in improving the functional movements that are performed with over $90^{\circ}$ arm elevations.

During active elevation of the arms on a scaption plane, the activity of the lower serratus anterior (LSA) gradually increases ${ }^{13)}$. In particular, this muscle's activity is maximized when the arms are elevated above $120^{\circ}$ under applied loads ${ }^{14)}$. In addition, the LSA provides a force couple to the lower scapular rotation coupled with the lower trapezius (LT), thereby reducing loading of the $\mathrm{UT}^{15}$. As this also clearly shows the functional importance of the LSA, recent studies have been conducted to identify the intramuscular activation of the SA during performance of various shoulder rehabilitation exercises ${ }^{11,16)}$.

Among the various PNF patterns, upper-extremity flexion, abduction, and external rotation patterns are the naturally used motions in activities of daily living when individuals perform tasks with their arms raised. Therefore, in clinical practice, such patterns are variously applied to patients with shoulder problems. Upper-extremity flexion, abduction and external rotation patterns can be indirectly applied by fixing the arms on the floor in a quadruped posi- 




Fig. 1. The start (A) and end positions (B) for the push-up plus exercise

tion and moving the trunk backward towards the heels. In this manner, the PNF exercise using upper-extremity patterns can apply the movements performed in activities of daily living with an over $90^{\circ}$ arm elevation as closed chain exercises and thus is likely to activate the LSA. Although frequently applied in clinical practice, studies that have identified the effects of PNF exercise are limited. In this context, this study was conducted to identify the effects of PNF and PUP exercises on the UT, LT, and MSA (middle serratus anterior), as well as the LSA activation and the UT/ SA ratios.

\section{SUBJECTS AND METHODS}

The study subjects were 14 healthy men who agreed to participate after receiving an explanation of the study procedure and objective. For study consistency, all study participants were right-handed. Individuals who experienced upper-extremity pain and discomfort, were incapable of doing exercise, or had a winging scapular, were excluded from this study.

Surface electromyography (EMG) with four channels was performed using an LXE 3204 (Laxtha Inc., Daejeon, Korea) with a bandwidth of $20-450 \mathrm{~Hz}$ and a sampling ratio of $1024 \mathrm{~Hz}$. In order to reduce skin resistance, the skin of the subjects was washed with alcohol and then shaved in the relevant area before attaching the surface electrodes. The electrodes were attached to the UT, LT, MSA and LSA. For the UT, an electrode was attached to the midpoint between the spinous process of the 7th cervical vertebra and the acromion. For the LT, it was attached to a point $1.5 \mathrm{~cm}$ lateral and obliquely to the T6 spinal process. For the MSA and LSA, electrodes were attached to the midaxillary line of the right side over the 5 th rib and the belly of the muscle located over the 7 th rib, respectively ${ }^{11,16)}$

A number of variables exist for the normalization of surface EMG amplitude during measurement of maximal voluntary isometric contraction (MVIC) for scapulothoracic muscles ${ }^{17)}$. Since resistance can be applied to the humerus during measurement of MVIC for trapezius and serratus anterior muscles, which subsequently causes muscle fatigue and strain, individual responses to a fixed load may provide a more stable base ${ }^{11)}$. Consequently, to normalize the surface EMG amplitude in this study, all subjects wore a $15-\mathrm{lb}$ sandbag on the right forearm and maintained a $90^{\circ}$ shoulder flexion on the scaption plane for $3 \mathrm{~s}$ while the reference voluntary isometric contraction (RVIC) was measured ${ }^{11)}$. Af-



Fig. 2. The start (A) and end positions (B) for the PNF exercise

ter 3 repetitions of this motion, their average was selected as an RVIC value. To prevent muscle fatigue, all participants had a 1-min break after performing each motion. Participants performed the PUP and PNF exercises in a quadruped position with their hands and knees placed vertically below their shoulder and hip joints. In the PUP exercise, the subjects maintained complete scapular protraction while completely extending their elbow joints from the floor (Fig. 1). For consistency of starting position, all subjects started the PNF exercise in the same position as the PUP exercise, and then diagonally lowered their right ischial tuberosity towards the opposite heel (left heel) while fixing their arms on the floor (Fig. 2). They also performed a diagonal motion until the right ischial tuberosity was located at the midline of the body. This was accomplished by marking the midline with tape. While the subjects moved their trunks backward diagonally, they applied a PNF upper-extremity pattern by pressing the right arm toward the floor in flexion, abduction and external rotation directions.

The surface EMG activity of all muscles was collected for $5 \mathrm{~s}$ while the subjects performed each exercise. The middle 3 seconds of collected data were used for analysis. Exercise in all subjects was controlled by having them perform each exercise for $10 \mathrm{~min}$ with auditory stimulation, and each exercise was randomly repeated three times. The subjects rested for $2 \mathrm{~min}$ after each exercise. The MannWhitney test was conducted to compare the muscle activity and the UT/SA ratios of the two exercises. For verification of statistical significance, the statistical significance level was set at $\alpha=0.05$. The collected data were analyzed using SPSS version 12.0 (SPSS Inc., Chicago, IL, USA).

\section{RESULTS}

The average age, height, weight and BMI of the subjects in this study were $23.14 \pm 2.48,176.14 \pm 5.55 \mathrm{~cm}, 66.14 \pm$ $6.35 \mathrm{~kg}$ and $21.31 \pm 1.63$, respectively. The activity of the scapular stabilizing muscles during the PUP and PNF exercises is shown in Table 1. While the UT activity showed no statistically significant difference between the two exercises, the LT activity increased to a significantly higher level during the PNF exercise as compared with the PUP exercise. In comparison, the MSA activity increased more, to a statistically significant level, during the PUP exercise than during the PNF exercise. On the other hand, the LSA activity increased more during the PNF exercise than during the PUP exercise, which was also statistically significant. The UT/SA ratios are shown in Table 2. While the UT/ 
Table 1. The normalized EMG data of the scapular stabilizing muscles during push-up plus and PNF exercises

\begin{tabular}{lcc}
\hline & Push-up plus & PNF exercise \\
\hline Upper trapezius & $15.23 \pm 3.88$ & $14.47 \pm 3.38$ \\
Lower trapezius* $^{*}$ & $19.02 \pm 5.14$ & $27.43 \pm 8.18$ \\
Middle serratus anterior $^{*}$ & $67.98 \pm 19.94$ & $52.55 \pm 14.54$ \\
Lower serratus anterior $^{*}$ & $58.14 \pm 16.78$ & $78.25 \pm 16.75$
\end{tabular}

Values are means \pm standard deviation (\%RVIC). *Significant difference $(\mathrm{p}<0.05)$

MSA ratio exhibited no differences between the two exercises, the UT/LSA ratio was significantly lower during the PNF exercise than during the PUP exercise.

\section{DISCUSSION}

In this study, the UT activity exhibited no statistically significant differences between the two groups. However, the LT activity was observed to be higher during the PNF exercise than during the PUP exercise, and the difference was statistically significant. The LT contributes to the upward rotation and posterior tilt of the scapular while reducing the impingement below the acromion during an arm elevation $^{3)}$. It has been reported that this muscle is likely to become more active in motions that require the scapular's dynamic stability rather than its static stability ${ }^{11)}$. The PNF exercise in this study indirectly increased the range of motion (ROM) of arm elevation through a posterior movement of the trunk. As a result, the demand for upward rotation and dynamic stability of the scapular is considered to have increased, which subsequently increased the LT activity to a higher level than during the PUP exercise. It is of note that a previous study that conducted a standard PUP exercise $^{11)}$ reported LT activity with an RVIC of about $13 \%$. In the present study, the LT activity indeed exhibited a higher level during the PNF exercise, which exhibited an RVIC of about $27 \%$. This indicates that the PNF exercise was more effective than the PUP exercise in strengthening the LT.

The LSA provides a couple force to the upward rotation of the lower scapular with the $\mathrm{LT}^{15}$. In addition, maximum upward rotation of the scapular occurs during an arm elevation of $80-140^{\circ 18)}$. In this study, the subjects gradually elevated their arms from $90^{\circ}$ to $140^{\circ}$ during the PNF exercise. As a result, the PNF exercise increased the LSA activity more in comparison with the PUP exercise, and the difference was statistically significant. The reason for these results is that the roles of both the LT, which provides a couple force to the continual upward rotation of the lower scapular, and the LSA increased. These results also support the findings of a previous study ${ }^{19}$ ) in which the increase in the ROM of arm elevation from $90^{\circ}$ to $140^{\circ}$ during a wall slide exercise resulted in a corresponding increase in the LSA activity.

In previous studies ${ }^{14,20)}$ that observed the SA activity in the same MSA area as targeted in the present study, the PUP exercise was reported to be a more effective exercise for strengthening the SA when compared with other shoulder rehabilitation exercises. The PUP exercise in this study
Table 2. The upper trapezius/serratus anterior ratios during push-up plus and PNF exercises

\begin{tabular}{ccc}
\hline & Push-up plus & PNF exercise \\
\hline UT/MSA & $0.25 \pm 0.10$ & $0.30 \pm 0.13$ \\
UT/LSA & $0.30 \pm 0.17$ & $0.20 \pm 0.07$ \\
\hline
\end{tabular}

*Significant difference $(\mathrm{p}<0.05)$. UT/MSA, upper trapezius/middle serratus anterior; UT/LSA, upper trapezius/lower serratus anterior

was also found to generate a significantly higher level of MSA muscle activity than the PNF exercise. Although the MSA muscle activity was significantly higher during the PUP exercise than during the PNF exercise, the UT/MSA ratio showed no statistically significant difference between the two. This is considered a result of the fact that the PNF exercise generated higher levels of LT and LSA activity than the PUP exercise, which, in turn, reduced the UT activity comparatively.

The UT/LSA ratio was significantly lower during the PNF exercise than during the PUP exercise. This may suggest that the PNF exercise can be a more effective training method for the shoulder rehabilitation of people with an unbalanced UT/LSA ratio. Furthermore, the PNF exercise heightened the activity of the LT and LSA, which are the muscles that make up the force couple that provides the upward rotation of the lower scapular, to a significantly higher level compared with the PUP exercise. This shows that the PNF exercise is more effective compared with the PUP exercise, in recovering the upward rotation and dynamic stability of the scapular, which are necessary for elevating the arms over $90^{\circ}$ in activities of daily living. Given that it provides the effects of traction to the shoulder joints by moving the trunk backward while keeping the arms fixed on the floor and pressing the hands toward the floor in a quadruped position, the PNF exercise is considered to help relieve the symptoms of shoulder impingement.

One limitation of the present study was that due to the absence of kinematic data, it could not accurately control the trunk movement or PNF upper-extremity pattern. In particular, there were limitations in maintaining consistent levels of arm pressure on the floor and the ROM of arm elevation during the PNF exercise. Therefore, while compensating for the present limitations, future research would need to identify the activation of scapular stabilizing muscles according to the ROM of arm elevation during the PNF exercise.

\section{REFERENCES}

1) Williams GR Jr, Shakil M, Klimkiewicz J, et al.: Anatomy of the scapulothoracic articulation. Clin Orthop Relat Res, 1999, 359: 237-246. [Medline] [CrossRef]

2) Lear LJ, Gross MT: An electromyographical analysis of the scapular stabilizing synergists during a push-up progression. J Orthop Sports Phys Ther, 1998, 28: 146-157. [Medline]

3) Ludewig PM, Cook TM, Nawoczenski DA: Three-dimensional scapular orientation and muscle activity at selected positions of humeral elevation. J Orthop Sports Phys Ther, 1996, 24: 57-65. [Medline]

4) Ludewig PM, Reynolds JF: The association of scapular kinematics and glenohumeral joint pathologies. J Orthop Sports Phys Ther, 2009, 39: 90-104. [Medline] 
5) Lukasiewicz AC, McClure P, Michener L, et al.: Comparison of 3-dimensional scapular position and orientation between subjects with and without shoulder impingement. J Orthop Sports Phys Ther, 1999, 29: 574-583. [Medline]

6) Glousman R, Jobe F, Tibone J, et al.: Dynamic electromyographic analysis of the throwing shoulder with glenohumeral instability. J Bone Joint Surg Am, 1988, 70: 220-226. [Medline]

7) Scovazzo ML, Browne A, Pink M, et al.: The painful shoulder during freestyle swimming. An electromyographic cinematographic analysis of twelve muscles. Am J Sports Med, 1991, 19: 577-582. [Medline] [CrossRef]

8) Ludewig PM, Cook TM: Alterations in shoulder kinematics and associated muscle activity in people with symptoms of shoulder impingement. Phys Ther, 2000, 80: 276-291. [Medline]

9) Ludewig PM, Hoff MS, Osowski EE, et al.: Relative balance of serratus anterior and upper trapezius muscle activity during push-up exercises. Am J Sports Med, 2004, 32: 484-493. [Medline] [CrossRef]

10) Maenhout A, Van Praet K, Pizzi L, et al.: Electromyographic analysis of knee push-up plus variations: what is the influence of the kinetic chain on scapular muscle activity? Br J Sports Med, 2010, 44: 1010-1015. [Medline] [CrossRef]

11) Park SY, Yoo WG: Differential activation of parts of the serratus anterio muscle during push-up variations on stable and unstable bases of support. J Electromyogr Kinesiol, 2011, 21: 861-867. [Medline] [CrossRef]

12) Lunden JB, Braman JP, Laprade RF, et al.: Shoulder kinematics during the wall push-up plus exercise. J Shoulder Elbow Surg, 2010, 19: 216-223. [Medline] [CrossRef]
13) Bagg SD, Forrest WJ: Electromyographic study of the scapular rotators during arm abduction in the scapular plane. Am J Phys Med, 1986, 65: 111-124. [Medline]

14) Moseley JB Jr, Jobe FW, Pink M, et al.: EMG analysis of the scapular muscles during a shoulder rehabilitation program. Am J Sports Med, 1992, 20: 128-134. [Medline] [CrossRef]

15) Inman VT, Saunders JB, Abbott LC: Observations of the function of the shoulder joint. J Bone Joint Surg, 1944, 26: 1-31.

16) Holtermann A, Mork PJ, Andersen LL, et al.: The use of EMG biofeedback for learning of selective activation of intra-muscular parts within the serratus anterior muscle: a novel approach for rehabilitation of scapular muscle imbalance. J Electromyogr Kinesiol, 2010, 20: 359-365. [Medline] [CrossRef]

17) Ekstrom RA, Soderberg GL, Donatelli RA: Normalization procedures using maximum voluntary isometric contractions for the serratus anterior and trapezius muscles during surface EMG analysis. J Electromyogr Kinesiol, 2005, 15: 418-428. [Medline] [CrossRef]

18) Bagg SD, Forrest WJ: A biomechanical analysis of scapular rotation during arm abduction in the scapular plane. Am J Phys Med Rehabil, 1988, 67: 238-245. [Medline]

19) Hardwick DH, Beebe JA, McDonnell MK, et al.: A comparison of serratus anterior muscle activation during a wall slide exercise and other traditional exercises. J Orthop Sports Phys Ther, 2006, 36: 903-910. [Medline] [CrossRef]

20) Decker MJ, Hintermeister RA, Faber KJ, et al.: Serratus anterior muscle activity during selected rehabilitation exercises. Am J Sports Med, 1999, 27: 784-791. [Medline] 